\title{
STOCHASTIC BOUNDARY CROSSING PROBABILITIES FOR THE BROWNIAN MOTION
}

\author{
XIAONAN CHE* AND \\ ANGELOS DASSIOS, ${ }^{* *}$ London School of Economics and Political Science
}

\begin{abstract}
Using martingale methods, we derive a set of theorems of boundary crossing probabilities for a Brownian motion with different kinds of stochastic boundaries, in particular compound Poisson process boundaries. We present both the numerical results and simulation experiments. The paper is motivated by limits on exposure of UK banks set by CHAPS. The central and participating banks are interested in the probability that the limits are exceeded. The problem can be reduced to the calculation of the boundary crossing probability from a Brownian motion with stochastic boundaries. Boundary crossing problems are also very popular in many fields of statistics.
\end{abstract}

Keywords: Boundary crossing probability; first passage time probability; Brownian motion

2010 Mathematics Subject Classification: Primary 60G40

Secondary 62P05; 60G51

\section{Introduction}

Let $\left\{X_{t}\right\}_{t \geq 0}$ be a continuous-time standard Brownian motion, and let two continuous functions $u(t)$ and $l(t)$ be the upper boundary and lower boundary, respectively. Define $\tau_{1}$ as the upper first passage time and $\tau_{2}$ as the lower first passage time:

$$
\tau_{1}=\inf _{t>0}\left\{X_{t} \geq u(t)\right\}, \quad \tau_{2}=\inf _{t>0}\left\{X_{t} \leq l(t)\right\} .
$$

Moreover, $\tau_{1}=\infty$ if $X_{t}<u(t)$ and $\tau_{2}=\infty$ if $X_{t}>l(t)$ for all $t>0$.

We are interested in the boundary crossing probabilities

$$
P_{u}^{(2)}=\mathbb{P}\left(\tau_{1}<\infty, \tau_{1}<\tau_{2}\right), \quad P_{l}^{(2)}=\mathbb{P}\left(\tau_{2}<\infty, \tau_{2}<\tau_{1}\right) .
$$

The first author to obtain an explicit representation for the distribution of the first passage time of a Brownian motion through linear boundaries was Doob; see [8, Appendix]. Anderson [2] derived formulae for closed regions using the reflection principle and inclusion-exclusion formulae. Escriba [9] solved the problem for sloping line boundaries. Scheike [20] derived an exact formula for broken linear boundaries.

Recently, simpler formulae and briefer derivations were obtained by Hall [11]. In the rest of the existing literature, some of the special cases of $P_{u}^{(2)}$ and $P_{l}^{(2)}$ are studied very well, mostly on piecewise-linear boundaries. The existing results are generally divided into two types according to two kinds of boundary. (i) The one-sided piecewise-linear boundary (in

Received 12 January 2012; revision received 23 August 2012.

* Postal address: Department of Statistics, London School of Economics and Political Science, Houghton Street, London, WC2A 2AE, UK.

** Email address: a.dassios@1se.ac.uk 
our notation, $u(t)$ is a piecewise-linear function and $l(t)=0$ for all $t$ ). The unconditional probability that a standard Brownian motion up-crosses a piecewise-linear boundary in a finite time interval [0,T] is obtained in [24] as $P_{u}^{(1)}(T)=\mathbb{P}\left(X_{t} \geq u(t), t<T\right)$, where $u(t)$ is a piecewise-linear polygonal function on the interval $[0, T]$. However, the calculation of $P_{u}^{(1)}(T)$ involves evaluation of a multiple integral, which cannot always be expressed by an explicit formula and, hence, must be solved numerically. In addition to this, Abundo [1] applied the time inversion property of Brownian motion and derived a simple and explicit formula for the conditional boundary crossing probability of $X_{t}$ with a piecewise-linear boundary, $u(t)$, consisting of two lines given that $X_{s}=\eta$ over a limit $[0, T]$. (ii) The two-sided boundary. Abundo [1] also solved the conditional crossing probability for $X_{t}$ with two symmetric linear boundaries, i.e. $P_{u}^{(2)}(T \mid \eta)=P_{l}^{(2)}(T \mid \eta)=\mathbb{P}\left(\left|X_{t}\right| \geq u(t), t<T \mid X_{s}=\eta\right)$, where $u(t)=$ $-l(t)=a+b t$ for all $t$. Novikov [13] derived a more general solution in terms of a piecewise function. Hence, the approximated boundary crossing probabilities $P_{\hat{u}}^{(2)}(T)$ and $P_{\hat{l}}^{(2)}(T)$ are found accordingly. Asymmetric linear boundaries have been studied recently, in particular, the probabilities $P_{l}^{(2)}$ and $P_{u}^{(2)}$ for time-dependent linear boundaries, i.e.

$$
c(t)= \begin{cases}b_{1}+c_{1} t, & b_{1}, c_{1}>0, \\ -b_{2}-c_{2} t, & b_{2}, c_{2}>0,\end{cases}
$$

are derived using powerful tools based on martingales [6], [7].

Calculations of this kind of probability are very popular in many fields of statistics. Such calculations have played an important role in many areas of statistical analysis [2], [5], [12], [16], [17], [19], [21], [22]. These boundary crossing probabilities have also attracted a lot of attention in finance. The problem of pricing so-called time-dependent barrier options can be reduced to the calculation of boundary crossing probabilities for a Brownian motion with a deterministic function [10], [14], [18]. Moreover, there are applications in other areas, such as biophysical models [15].

This paper is particularly motivated by an innovative development in banking systems, the large-valued interbank payment system (which is called the real-time gross settlement payment system and is known as CHAPS in the UK). In this particular payment system, the participating banks are concerned about credit risk, and wish to prevent the considerable credit exposure between two banks. (Although RTGS had replaced the Netting settlement system around the world, by the end of 2006, 93 of world's 174 central banks were already using RTGS systems [3], the possibility of unexpected exposures still exists.) There is evidence that suggests that in CHAPS, banks usually set bilateral (or multilateral) limits on the exposed position with others, and the limits are adjusted during the course of the business day under certain circumstances. Both the participating banks and the central bank are interested in the probabilities that these limits are exceeded. Previous studies have shown that Markov-type models are adequate for the UK real-time gross settlement system [6]. For a single bank, namely bank $A$, let the standard Brownian motion $X_{t}$ be the net balance between bank $A$ and bank $i$ at time $t$. Meanwhile, $u_{i}(t)$ is the bilateral limit set up by bank $A$ for bank $i$. In practice, as individual banks are very sensitive to their liquidity position when making risky transactions, once a predefined limit is reached, it is highly likely that the limit will continue increasing throughout the day over different occasions. Therefore, this problem can be reduced to the calculation of the boundary crossing probability for Brownian motion with stochastic boundaries. This approach can be adopted by both the policy maker in the central bank and the credit control departments of participating banks to lay down decisive actions. 
In this paper we aim to find the exact boundary crossing probability of standard Brownian motion with stochastic boundaries when the process can go without limit. In Section 2, the most important theorems of the paper are stated, and we provide a method to derive the boundary crossing probability for standard Brownian motion with stochastic boundaries. Powerful tools such as martingales and the infinitesimal generator of Brownian motion are used. Our approach is based on the classical martingale stopping approach described in [4] and elsewhere (see, for example, [23]). The proposed approach allows us to obtain a set of simple formulae for the two-sided boundaries crossing probabilities. Additionally, simulation results are given. In Section 3 we draw our conclusions.

\section{Stochastic boundaries}

This section contains the most important theorems of this paper.

\subsection{General stochastic boundaries}

Consider a standard Brownian motion $\left\{X_{t}\right\}_{t \geq 0}$ which initially starts from $x_{0}$, with boundaries such that $Z_{t}=\sum_{i=1}^{N(t)} Y_{i}+b$, where $N(t)$ is a Poisson process with rate $\lambda$ and the jump size $Y_{i}>0$ has distribution function $H(y)$ and is independent of the Brownian motion. Define the first passage time as $\tau=\inf _{t>0}\left\{t:\left|X_{t}\right|=Z_{t}\right\}$, the first time that $X_{t}$ exceeds one of the two boundaries, with the convention that $\tau=\infty$ if $\left|X_{t}\right|<Z_{t}$ for all $t>0$.

Since the standard Brownian motion $X_{t}$ and Poisson process $Z_{t}$ are independent, the martingale that we are interested in is $\mathrm{e}^{-\gamma Z_{t}}\left(\mathrm{e}^{k X_{t}}+\mathrm{e}^{-k X_{t}}\right)$, where $\gamma$ and $k$ are nonnegative constants. This is true because the martingale for the Brownian motion $X_{t}$ is $\mathrm{e}^{-k^{2} t / 2}\left(\mathrm{e}^{k X_{t}}+\mathrm{e}^{-k X_{t}}\right)$, and, for the Poisson process, it is $\mathrm{e}^{-\gamma Z_{t}-\lambda t(\varphi(\gamma)-1)}$, where $\varphi(\gamma)=$ $\int_{0}^{\infty} \mathrm{e}^{-\gamma y} \mathrm{~d} H(y)$ is the Laplace transform of the distribution function of $Y_{i}$. One can find that $\lambda(1-\varphi(\gamma))=\frac{1}{2} k^{2}$; note that the two values of $k$ will yield identical martingales, so we will let $k=\sqrt{2 \lambda(1-\varphi(\gamma))}$.

Owing to the martingale property and Doob's optional stopping theorem, we obtain

$$
\begin{aligned}
& \mathbb{E}\left[\mathrm{e}^{-\gamma Z_{t}}\left(\mathrm{e}^{k X_{t}}+\mathrm{e}^{-k X_{t}}\right) \mathbf{1}_{\{\tau<t\}}\right]+\mathbb{E}\left[\mathrm{e}^{-\gamma Z_{t}}\left(\mathrm{e}^{k X_{t}}+\mathrm{e}^{-k X_{t}}\right) \mathbf{1}_{\{\tau \geq t\}}\right] \\
& =\mathbb{E}\left[\mathrm{e}^{-\gamma Z_{0}}\left(\mathrm{e}^{k X_{0}}+\mathrm{e}^{-k X_{0}}\right)\right], \\
& \mathbb{E}\left[\mathrm{e}^{-\gamma Z_{\tau}}\left(\mathrm{e}^{k X_{\tau}}+\mathrm{e}^{-k X_{\tau}}\right) \mathbf{1}_{\{\tau<\infty\}}\right]=\mathrm{e}^{-\gamma b}\left(\mathrm{e}^{k x_{0}}+\mathrm{e}^{-k x_{0}}\right),
\end{aligned}
$$

where $\mathbf{1}_{\{\tau<t\}}$ and $\mathbf{1}_{\{\tau \geq t\}}$ are indicator functions.

By the strong law of large numbers for Brownian motion, $\lim _{t \rightarrow \infty} X_{t} / t \rightarrow 0$. Then the second term on the left-hand side of (1) becomes

$$
\begin{aligned}
\lim _{t \rightarrow \infty} & \mathbb{E}\left[\mathrm{e}^{-\gamma Z_{t}}\left(\mathrm{e}^{k X_{t}}+\mathrm{e}^{-k X_{t}}\right) \mathbf{1}_{\{\tau \geq t\}}\right] \\
& =\mathbb{E}\left[\lim _{t \rightarrow \infty} \mathrm{e}^{-\gamma Z_{t}+k X_{t}} \mathbf{1}_{\{\tau \geq t\}}+\lim _{t \rightarrow \infty} \mathrm{e}^{-\gamma Z_{t}-k X_{t}} \mathbf{1}_{\{\tau \geq t\}}\right] \\
& =\mathbb{E}\left[\mathrm{e}^{\lim _{t \rightarrow \infty}-\gamma Z_{t}} \mathrm{e}^{\lim _{t \rightarrow \infty} t k X_{t} / t} \mathbf{1}_{\{\tau=\infty\}}\right]+\mathbb{E}\left[\mathrm{e}^{\lim _{t \rightarrow \infty}-\gamma Z_{t}} \mathrm{e}^{\lim _{t \rightarrow \infty}-t k X_{t} / t} \mathbf{1}_{\{\tau=\infty\}}\right] \\
& =0 .
\end{aligned}
$$

Hence, (2) follows immediately.

We start with the simple case where the two stochastic boundaries are symmetric about the time horizon. Thus, consider the following set of equations only: for $j=0,1,2, \ldots$,

$$
\begin{aligned}
\mathbb{E}\left[\mathrm{e}^{-\gamma_{j} Z_{\tau}}\left(\mathrm{e}^{k_{j} X_{\tau}}+\mathrm{e}^{-k_{j} X_{\tau}}\right) \mathbf{1}_{\{\tau<\infty\}}\right]=\mathrm{e}^{-\gamma_{j} z_{0}}\left(\mathrm{e}^{k_{j} x_{0}}+\mathrm{e}^{-k_{j} x_{0}}\right), \\
\mathbb{E}\left[\mathrm{e}^{-\gamma_{j+1} Z_{\tau}}\left(\mathrm{e}^{k_{j+1} X_{\tau}}+\mathrm{e}^{-k_{j+1} X_{\tau}}\right) \mathbf{1}_{\{\tau<\infty\}}\right]=\mathrm{e}^{-\gamma_{j+1} z_{0}}\left(\mathrm{e}^{k_{j+1} x_{0}}+\mathrm{e}^{-k_{j+1} x_{0}}\right) .
\end{aligned}
$$


As $k_{j}=\sqrt{2 \lambda\left(1-\varphi\left(\gamma_{j}\right)\right)}$, we have

$$
\begin{aligned}
& \mathbb{E}\left[\mathrm{e}^{-\left(\gamma_{j}-\sqrt{2 \lambda\left(1-\varphi\left(\gamma_{j}\right)\right)}\right) Z_{\tau}} \mathbf{1}_{\{\tau<\infty\}}\right]+\mathbb{E}\left[\mathrm{e}^{-\left(\gamma_{j}+\sqrt{2 \lambda\left(1-\varphi\left(\gamma_{j}\right)\right)}\right) Z_{\tau}} \mathbf{1}_{\{\tau<\infty\}}\right] \\
& \quad=\mathrm{e}^{-\gamma_{j} b}\left(\mathrm{e}^{k_{j} x_{0}}+\mathrm{e}^{-k_{j} x_{0}}\right), \\
& \mathbb{E}\left[\mathrm{e}^{-\left(\gamma_{j+1}-\sqrt{2 \lambda\left(1-\varphi\left(\gamma_{j+1}\right)\right)}\right)} Z_{\tau} \mathbf{1}_{\{\tau<\infty\}}\right]+\mathbb{E}\left[\mathrm{e}^{-\left(\gamma_{j+1}+\sqrt{2 \lambda\left(1-\varphi\left(\gamma_{j+1}\right)\right)}\right) Z_{\tau}} \mathbf{1}_{\{\tau<\infty\}}\right] \\
& \quad=\mathrm{e}^{-\gamma_{j+1} b}\left(\mathrm{e}^{k_{j+1} x_{0}}+\mathrm{e}^{-k_{j+1} x_{0}}\right) .
\end{aligned}
$$

In (3) and (4), we choose $\gamma_{j}$ such that

$$
\gamma_{j}+\sqrt{2 \lambda\left(1-\varphi\left(\gamma_{j}\right)\right)}=\gamma_{j+1}-\sqrt{2 \lambda\left(1-\varphi\left(\gamma_{j+1}\right)\right)},
$$

so when subtracting (3) from (4) for $j=0,2,4, \ldots$, we obtain

$$
\begin{gathered}
\mathbb{E}\left[\mathrm{e}^{-\left(\gamma_{j}-\sqrt{2 \lambda\left(1-\varphi\left(\gamma_{j}\right)\right)}\right) Z_{\tau}} \mathbf{1}_{\{\tau<\infty\}}\right]-\mathbb{E}\left[\mathrm{e}^{-\left(\gamma_{j+1}+\sqrt{2 \lambda\left(1-\varphi\left(\gamma_{j+1}\right)\right)}\right) Z_{\tau}} \mathbf{1}_{\{\tau<\infty\}}\right] \\
=\mathrm{e}^{-\gamma_{j} b}\left(\mathrm{e}^{k_{j} x_{0}}+\mathrm{e}^{-k_{j} x_{0}}\right)-\mathrm{e}^{-\gamma_{j+1} b}\left(\mathrm{e}^{k_{j+1} x_{0}}+\mathrm{e}^{-k_{j+1} x_{0}}\right) .
\end{gathered}
$$

Using (5), when summing (6) over $j$, we have

$$
\begin{gathered}
\mathbb{E}\left[\mathrm{e}^{-\left(\gamma_{0}-\sqrt{2 \lambda\left(1-\varphi\left(\gamma_{0}\right)\right)}\right) Z_{\tau}} \mathbf{1}_{\{\tau<\infty\}}\right]-\mathbb{E}\left[\mathrm{e}^{-\left(\gamma_{n}+\sqrt{2 \lambda\left(1-\varphi\left(\gamma_{n}\right)\right)}\right) Z_{\tau}} \mathbf{1}_{\{\tau<\infty\}}\right] \\
=\sum_{j=0}^{n}(-1)^{j} \mathrm{e}^{-\gamma_{j} b}\left(\mathrm{e}^{k_{j} x_{0}}+\mathrm{e}^{-k_{j} x_{0}}\right) .
\end{gathered}
$$

Now let $\theta_{0}=\gamma_{0}-\sqrt{2 \lambda\left(1-\varphi\left(\gamma_{0}\right)\right)}$. Then

$$
\theta_{n}=\gamma_{n}-\sqrt{2 \lambda\left(1-\varphi\left(\gamma_{n}\right)\right)} \quad \text { and } \quad \gamma_{n}+\sqrt{2 \lambda\left(1-\varphi\left(\gamma_{n}\right)\right)}=2 \gamma_{n}-\theta_{n} .
$$

Then Theorem 1 below follows.

Theorem 1. Let $\varphi(\gamma)$ be the Laplace transform of the distribution function of $Y_{i}$. Then the probability that $X_{t}$ exceeds any one of the two boundaries $Z_{t}$ and $-Z_{t}$ can be obtained via

$$
\mathbb{E}\left[\mathrm{e}^{-\theta_{0} Z_{\tau}} \mathbf{1}_{\{\tau<\infty\}}\right]=\mathbb{E}\left[\mathrm{e}^{-\left(2 \gamma_{n}-\theta_{n}\right) Z_{\tau}} \mathbf{1}_{\{\tau<\infty\}}\right]+\sum_{j=0}^{n}(-1)^{j} \mathrm{e}^{-\gamma_{j} b}\left(\mathrm{e}^{k_{j} x_{0}}+\mathrm{e}^{-k_{j} x_{0}}\right)
$$

by letting $\theta_{0}=0$ and $n \rightarrow \infty$, where

$$
\theta_{0}=\gamma_{0}-\sqrt{2 \lambda\left(1-\varphi\left(\gamma_{0}\right)\right)} \text { and } \theta_{n}=\gamma_{n}-\sqrt{2 \lambda\left(1-\varphi\left(\gamma_{n}\right)\right)}
$$

\subsection{Boundaries with exponential jumps}

When the jumps of a compound Poisson process have an exponential distribution, the twosided boundary crossing probabilities $\mathbb{P}^{(2)}(\tau<\infty)$ can be derived explicitly.

Theorem 2. Suppose that a standard Brownian motion $\left\{X_{t}\right\}_{t \geq 0}$ with boundaries that are a compound Poisson process, such as $Z_{t}=\sum_{i=1}^{N(t)} Y_{i}+b$, and exponential jump sizes $Y_{i}$, initially starts from 0 . The first passage time probability is

$$
\mathbb{P}^{(2)}(\tau<\infty)=2 \sum_{j=0}^{\infty}(-1)^{j} \mathrm{e}^{-\gamma_{j} b}
$$


where $\gamma_{j}=\alpha k_{j}^{2} /\left(2 \lambda-k_{j}^{2}\right)$ and the $k_{j}$ s are satisfied by

$$
\left(2 \lambda-k_{j}^{2}\right) k_{j+1}^{2}+2 \lambda \alpha k_{j+1}-2 \lambda \alpha k_{j}+2 \lambda k_{j}^{2}-4 \lambda^{2}=0
$$

with $k_{0}=\left(\sqrt{\alpha^{2}+8 \lambda}-\alpha\right) / 2$, where $\lambda$ and $1 / \alpha$ are the rate of the Poisson process and the mean of the exponential jumps, respectively.

Proof. The proof is a modification of the proof of Theorem 1. When the jump size $Y_{i}$ is exponentially distributed with parameter $\alpha$, the function $\varphi\left(\gamma_{j}\right)=\int_{0}^{\infty} \mathrm{e}^{-\gamma_{j} y} \alpha \mathrm{e}^{-\alpha y} \mathrm{~d} y=$ $\alpha /\left(\alpha+\gamma_{j}\right)$. Hence, the constants of the martingale are $k_{j}=\sqrt{2 \lambda \gamma_{j} /\left(\alpha+\gamma_{j}\right)}$ and $\gamma_{j}=$ $\alpha k_{j}^{2} /\left(2 \lambda-k_{j}^{2}\right)$. Similarly, we want $k_{j}$ to satisfy (5), which means that

$$
\frac{\alpha k_{j}^{2}}{2 \lambda-k_{j}^{2}}+k_{j}=\frac{\alpha k_{j+1}^{2}}{2 \lambda-k_{j+1}^{2}}-k_{j+1}
$$

yielding (9); hence, the $k_{j}$ s have the recurrence relation

$$
k_{j+1}=\frac{\sqrt{\lambda^{2} \alpha^{2}+2 \lambda\left(2 \lambda-k_{j}^{2}\right)\left(\alpha k_{j}-k_{j}^{2}+2 \lambda\right)}-\alpha \lambda}{2 \lambda-k_{j}^{2}} .
$$

Moreover,

$$
\theta_{j}=\frac{\alpha k_{j}^{2}}{2 \lambda-k_{j}^{2}}-k_{j}=\frac{k_{j}\left(k_{j}^{2}+\alpha k_{j}-2 \lambda\right)}{2 \lambda-k_{j}^{2}}
$$

in (7). By letting $\theta_{0}=0$, the initial value $k_{0}$ can be found in terms of $\lambda$ and $\alpha$ only, i.e. $k_{0}=\left(\sqrt{\alpha^{2}+8 \lambda}-\alpha\right) / 2$, since both the rates $\alpha$ and $\lambda$ are nonnegative. Thereby, (7) becomes

$$
\mathbb{P}(\tau<\infty)=\mathbb{E}\left[\mathrm{e}^{-\left(2 \gamma_{n}-\theta_{n}\right) Z_{\tau}} \mathbf{1}_{\{\tau<\infty\}}\right]+\sum_{j=0}^{n}(-1)^{j} \mathrm{e}^{-\gamma_{j} b}\left(\mathrm{e}^{k_{j} x_{0}}+\mathrm{e}^{-k_{0} x_{0}}\right) .
$$

Since we are concerned with the boundary crossing probability over an infinite time interval, to the extent of Theorem 1, (9) can be rewritten as

$$
\begin{gathered}
\left(2 \lambda-k_{j}^{2}\right)\left(k_{j+1}^{2}-k_{j}^{2}\right)+2 \lambda \alpha\left(k_{j+1}-k_{j}\right)-\left(2 \lambda-k_{j}^{2}\right)^{2}=0 \\
\Longrightarrow \quad\left(2 \lambda-k_{j}^{2}\right)\left(k_{j+1}^{2}-2 \lambda\right)+2 \lambda \alpha\left(k_{j+1}-k_{j}\right)=0 .
\end{gathered}
$$

Define $\lim _{j \rightarrow \infty} k_{j}=k$. Due to (11), we obtain $\lim _{j \rightarrow \infty} k_{j}=\sqrt{2 \lambda}$, with both $\gamma_{j}$ and $\theta_{j}$ monotonic functions. Then (10) becomes

$$
\mathbb{P}(\tau<\infty)=\sum_{j=0}^{\infty} 2(-1)^{j} \mathrm{e}^{-\gamma_{j} b}
$$

with the assumption that the process starts from 0 , i.e. $X_{0}=0$. 
2.2.1. Numerical solutions. To illustrate the use of Theorem 2 , we calculate first passage time probabilities using (8) for $\lambda=0.05,0.1,1$ and $\alpha=\frac{1}{50}, \frac{1}{20}, \frac{1}{10}$, with eight different starting positions of the boundaries, $b=1,2,3,4,5,10,15$, 20; see Table 1 . We see that the first passage time probabilities would reach the tabulated values very quickly (the smaller the value of $b$, the more iterations are required, but at most 16 iterations are sufficient), i.e. in (8),

$$
\mathbb{P}(\tau<\infty)=2 \sum_{j=0}^{\infty}(-1)^{j} \mathrm{e}^{-\gamma_{j} b} \approx 2 \sum_{j=0}^{16}(-1)^{j} \mathrm{e}^{-\gamma_{j} b} .
$$

Meanwhile, in the course of the experiment, the values of $k_{j}$ (see Table 2 for examples) which are varied by $\lambda$, the rate of the Poisson process, converge particularly fast.

On the other hand, the values of both $\theta_{j}$ and $\gamma_{j}$ (Table 2) are strictly increasing. Although, it has not been shown in the tables, if we keep running the experiment for more iterations $(j>16, j \rightarrow \infty)$, both $\theta_{j}$ and $\gamma_{j}$ tend to $\infty$ numerically, as does $2 \gamma_{j}-\theta_{j}$. Hence, the term $\mathbb{E}\left[\mathrm{e}^{-\left(2 \gamma_{n}-\theta_{n}\right) Z_{\tau}} \mathbf{1}_{\{\tau<\infty\}}\right]$ in (10) will equal 0 for large $n$.

We illustrate the results of Table 1 in Figure 1, where the horizontal axis is the value of $b$ and the vertical axis represents the probabilities. In Figure 1, the plots are separated into different groups. In each group, the three plots have $\lambda$ in common and $\alpha$, the lighter the color of the curve, the larger the value of $\alpha$. Within groups, from $b=0$ till the point where the curves reach the maximum, they do not diverge away from each other; after the peak, the probabilities with the same $\lambda$ tend to separate; nevertheless, a confluence is achieved at the end (i.e. $b=20$ ). Among groups of curves, although the maximums occur over a short interval of $b$, the order can still be observed. The curves show an early peak and are then decreasing. Moreover, when the frequency of changes for the boundaries grows, the difference between the first passage time probabilities becomes less visible. To conclude, Figure 1 indicates that, in comparison with the effect of the value of $\lambda$, the rate of the Poisson process, the values of the mean size of the

TABLE 1: First passage time probabilities for various values of $\lambda, \alpha$, and $b$.

\begin{tabular}{rrrcc}
\hline \multirow{2}{*}{$\alpha$} & $b$ & \multicolumn{3}{c}{$\lambda$} \\
\cline { 3 - 5 } & & 0.05 & 0.1 & 1 \\
\hline$\frac{1}{50}$ & 0 & 0.9557 & 0.9124 & 0.4633 \\
& 5 & 0.4135 & 0.2219 & 0.0018 \\
& 10 & 0.0932 & 0.0252 & $1.59 \times 10^{-6}$ \\
& 15 & 0.0202 & 0.0028 & $1.42 \times 10^{-9}$ \\
& 20 & 0.0044 & 0.0003 & $1.27 \times 10^{-12}$ \\
\hline$\frac{1}{20}$ & 0 & 0.9609 & 0.9191 & 0.4697 \\
& 5 & 0.4419 & 0.2382 & 0.0019 \\
& 10 & 0.1074 & 0.0291 & $1.85 \times 10^{-6}$ \\
& 15 & 0.0250 & 0.0035 & $1.78 \times 10^{-9}$ \\
& 20 & 0.0058 & 0.0004 & $1.71 \times 10^{-12}$ \\
\hline$\frac{1}{10}$ & 0 & 0.9685 & 0.9295 & 0.4803 \\
& 5 & 0.4894 & 0.2665 & 0.0022 \\
& 10 & 0.1338 & 0.0366 & $2.36 \times 10^{-6}$ \\
& 15 & 0.0348 & 0.0050 & $2.56 \times 10^{-9}$ \\
& 20 & 0.0090 & 0.0006 & $2.78 \times 10^{-12}$ \\
\hline
\end{tabular}


TABLE 2: Values of $k_{j}, \theta_{j}$, and $\gamma_{j}$ when $\lambda=0.05$ and $\alpha=\frac{1}{50}, \frac{1}{20}$.

\begin{tabular}{|c|c|c|c|c|c|c|c|}
\hline \multicolumn{4}{|c|}{$\alpha=\frac{1}{50}$} & \multicolumn{4}{|c|}{$\alpha=\frac{1}{20}$} \\
\hline$j$ & $k_{j}$ & $\theta_{j}$ & $\gamma_{j}$ & $j$ & $k_{j}$ & $\theta_{j}$ & $\gamma_{j}$ \\
\hline 0 & 0.3064 & 0 & 0.3064 & 0 & 0.2922 & 0 & 0.2922 \\
\hline 1 & 0.3129 & 0.6128 & 0.9256 & 1 & 0.3077 & 0.5844 & 0.8922 \\
\hline 2 & 0.3142 & 1.2385 & 1.5527 & 2 & 0.3111 & 1.1999 & 1.511 \\
\hline 3 & 0.3148 & 1.8669 & 2.1817 & 3 & 0.3126 & 1.8221 & 2.1347 \\
\hline 4 & 0.3151 & 2.4965 & 2.8116 & 4 & 0.3134 & 2.4473 & 2.7607 \\
\hline 5 & 0.3153 & 3.1267 & 3.4420 & 5 & 0.3139 & 3.0741 & 3.388 \\
\hline 6 & 0.3155 & 3.7573 & 4.0728 & 6 & 0.3143 & 3.7019 & 4.0162 \\
\hline 7 & 0.3156 & 4.3883 & 4.7038 & 7 & 0.3145 & 4.3305 & 4.645 \\
\hline 8 & 0.3156 & 5.0194 & 5.3350 & 8 & 0.3147 & 4.9596 & 5.2743 \\
\hline 9 & 0.3157 & 5.6506 & 5.9663 & 9 & 0.3149 & 5.5891 & 5.9039 \\
\hline 10 & 0.3157 & 6.2820 & 6.5978 & 10 & 0.3150 & 6.2188 & 6.5339 \\
\hline 11 & 0.3158 & 6.9135 & 7.2293 & 11 & 0.3151 & 6.8489 & 7.164 \\
\hline 12 & 0.3158 & 7.5451 & 7.8609 & 12 & 0.3152 & 7.4792 & 7.7944 \\
\hline 13 & 0.3159 & 8.1768 & 0.4926 & 13 & 0.3153 & 8.1096 & 8.4249 \\
\hline 14 & 0.3159 & 8.8085 & 9.1244 & 14 & 0.3154 & 8.7402 & 9.0555 \\
\hline 15 & 0.3159 & 9.4402 & 9.7562 & 15 & 0.3154 & 9.3709 & 9.6863 \\
\hline
\end{tabular}

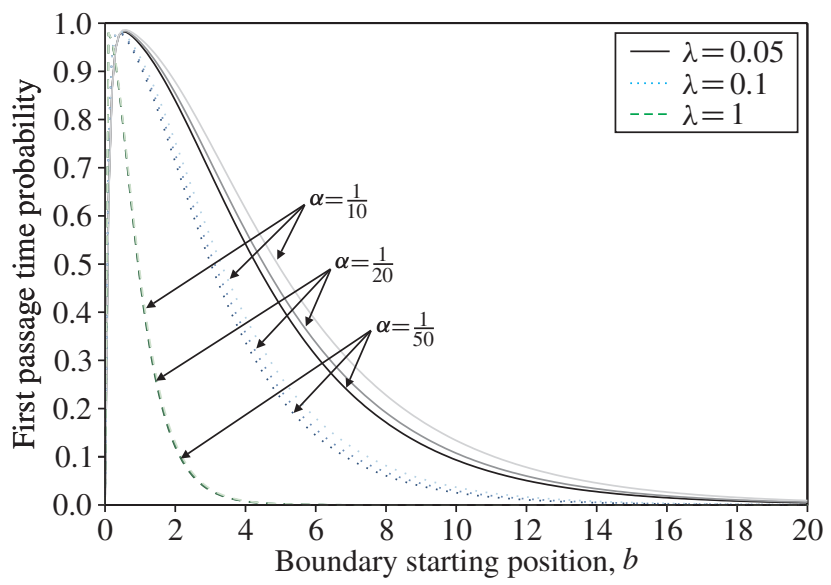

FIGURE 1: First passage time probabilities for various values of $\lambda, \alpha$, and $b$.

exponential jumps, $\alpha$, vary the probabilities over a more narrow interval. Last but not least, the initial value of the boundaries is the most deterministic factor of the crossing probabilities. When the boundaries initially start from $b \geq 20$, they are less likely to be crossed.

2.2.2. Monte Carlo simulation results. In this section we provide the corresponding simulated results. A discrete-time standard Brownian motion is generated using the Monte Carlo Markov chain, and the two symmetric compound Poisson processes with exponential changes are simulated in the same time horizon. We investigated the number of times the standard Brownian motion meets up with one of the boundaries over 1000 simulations. The results were repeated 100 times and the averaged probabilities and associated variances presented in Table 3 . 
TABLE 3: Simulated mean probabilities and their variances (in parentheses).

\begin{tabular}{rrccl}
\hline \multirow{2}{*}{$\alpha$} & $b$ & \multicolumn{3}{c}{$\lambda$} \\
\cline { 3 - 5 } & & 0.05 & 0.1 & \multicolumn{1}{c}{1} \\
\hline$\frac{1}{50}$ & 1 & $0.9554(0.000046)$ & $0.9141(0.000088)$ & $0.4633(0.000224)$ \\
& 5 & $0.4123(0.000256)$ & $0.2269(0.000166)$ & $0.0020(0.000002)$ \\
& 10 & $0.0968(0.000083)$ & $0.0264(0.000032)$ & $0.0000(0.000000)$ \\
& 15 & $0.0274(0.000026)$ & $0.0029(0.000003)$ & $0(0)$ \\
& 20 & $0.0041(0.000004)$ & $0.0003(0.000000)$ & $0(0)$ \\
\hline$\frac{1}{20}$ & 1 & $0.9637(0.000038)$ & $0.9172(0.000068)$ & $0.4645(0.000269)$ \\
& 5 & $0.4572(0.000234)$ & $0.2381(0.000148)$ & $0.0013(0.000001)$ \\
& 10 & $0.1037(0.000116)$ & $0.0310(0.000027)$ & $0.0000(0.000000)$ \\
& 15 & $0.0267(0.000026)$ & $0.0036(0.000004)$ & $0(0)$ \\
& 20 & $0.0067(0.000007)$ & $0.0009(0.000001)$ & $0(0)$ \\
\hline$\frac{1}{10}$ & 1 & $0.9665(0.000031)$ & $0.9272(0.000056)$ & $0.4868(0.000237)$ \\
& 5 & $0.4904(0.000187)$ & $0.2610(0.000227)$ & $0.0028(0.000003)$ \\
& 10 & $0.1328(0.000132)$ & $0.0347(0.000039)$ & $0.0000(0.000000)$ \\
& 15 & $0.0364(0.000037)$ & $0.0057(0.000007)$ & $0(0)$ \\
& 20 & $0.0090(0.000008)$ & $0.0009(0.000001)$ & $0(0)$ \\
\hline
\end{tabular}

For different values of $b$, the interval needs to be calculated at different levels for the simulated discrete standard Brownian motion. However, generally speaking, the results are closely equal to those in Table 1.

\subsection{Boundaries with constant jumps}

When the size of the jumps of the compound Poisson processes is constant, our methods can be applied immediately.

Corollary 1. Let $\left\{X_{t}\right\}_{t \geq 0}$ be a standard Brownian motion with boundaries such that $Z_{t}=$ $b+c N_{t}$, where $N_{t}$ is a Poisson process with parameter $\lambda$ and $c$ is a positive constant. The first passage time probability is given by

$$
\mathbb{P}(\tau<\infty)=2 \sum_{j=0}^{\infty}(-1)^{j} \mathrm{e}^{-\gamma_{j} b},
$$

where $\gamma_{j}=2 \lambda c /\left(2 \lambda-k_{j}^{2}\right)$ and the $k_{j}$ s satisfy the equation

$$
\left(2 \lambda-k_{j}^{2}\right) k_{j+1}^{2}+2 \lambda c k_{j+1}-2 \lambda c k_{j}+2 \lambda k_{j}^{2}-4 \lambda^{2}=0
$$

with $\lambda$ the rate of the Poisson process and $k_{0}^{(r)}$ the positive real solution of the equation

$$
k_{0}^{3}-2 \lambda k_{0}+2 \lambda c=0 .
$$

Proof. In this case, $\varphi(\gamma)=c / \gamma$, where $\varphi(\gamma)$ is defined in Theorem 1 . Therefore, $k_{j}=$ $\sqrt{2 \lambda\left(1-c / \gamma_{j}\right)}$ and $\gamma_{j}=2 \lambda c /\left(2 \lambda-k_{j}^{2}\right)$. Similarly to (5),

$$
\frac{2 \lambda c}{2 \lambda-k_{j}^{2}}+k_{j}=\frac{2 \lambda c}{2 \lambda-k_{j+1}^{2}}-k_{j+1} \text {. }
$$


TABLE 4: First passage time probabilities for various values of $\lambda, c$, and $b$.

\begin{tabular}{rrccc}
\hline & & \multicolumn{3}{c}{$\lambda$} \\
\cline { 3 - 5 } & & 0.05 & 0.1 & 1 \\
\hline$\frac{1}{50}$ & 1 & 0.9542 & 0.9125 & 0.4634 \\
& 5 & 0.4148 & 0.2224 & 0.0018 \\
& 10 & 0.0939 & 0.0253 & $1.60 \times 10^{-6}$ \\
& 15 & 0.0204 & 0.0029 & $1.43 \times 10^{-9}$ \\
& 20 & 0.0044 & 0.0003 & $1.27 \times 10^{-12}$ \\
\hline$\frac{1}{20}$ & 1 & 0.9610 & 0.9206 & 0.4701 \\
& 5 & 0.4520 & 0.2419 & 0.0019 \\
& 10 & 0.1126 & 0.0301 & $1.87 \times 10^{-6}$ \\
& 15 & 0.0268 & 0.0037 & $1.80 \times 10^{-9}$ \\
& 20 & 0.0064 & 0.0005 & $1.74 \times 10^{-12}$ \\
\hline$\frac{1}{10}$ & 1 & 0.9766 & 0.9368 & 0.4820 \\
& 5 & 0.5568 & 0.2872 & 0.0022 \\
& 10 & 0.1764 & 0.0427 & $2.45 \times 10^{-6}$ \\
& 15 & 0.0527 & 0.0062 & $2.71 \times 10^{-9}$ \\
& 20 & 0.0157 & 0.0009 & $3.00 \times 10^{-12}$ \\
\hline
\end{tabular}

Then $k_{j+1}$ can be found by solving the equation

$$
\left(2 \lambda-k_{j}^{2}\right) k_{j+1}^{2}+2 \lambda c k_{j+1}-2 \lambda c k_{j}+2 \lambda k_{j}^{2}-4 \lambda^{2}=0 .
$$

We note that this is (9) with $\alpha$ replaced by $c$ as in Theorem 2 . However, the initial value $k_{0}$ has a different expression in terms of $\lambda$ and $c$. We can derive $k_{0}$ from $\gamma_{0}-k_{0}=0$, i.e.

$$
k_{0}^{3}-2 \lambda k_{0}+2 \lambda c=0
$$

which has three roots, in general, with only the real root $k_{0}^{(r)}$ yielding convergent $k_{j}$. Moreover, if the values of the parameters $\lambda$ and $c$ have been chosen such that the discriminant $\Delta_{k}>0$, all the solutions of the cubic equation (13) are distinctly real; however, only one of them will lead to the convergence of $k_{j}$.

2.3.1. Numerical solutions. Similarly as in Section 2.2.1, in Table 4 we present the boundary crossing probabilities for a standard Brownian motion with symmetric compound Poisson boundaries having constant jumps, according to (12). We have taken the same values for the parameters: $\lambda=0.05,0.1,1, b=1,2,3,4,5,10,15,20$, and $c$ replacing $\alpha$ equal to $\frac{1}{50}, \frac{1}{20}$, and $\frac{1}{10}$.

A similar conclusion could be drawn as in the previous exponential jumps case, but with fewer iterations as the tabulated boundary crossing probabilities are achieved rapidly:

$$
\mathbb{P}(\tau<\infty)=2 \sum_{j=0}^{\infty}(-1)^{j} \mathrm{e}^{-\gamma_{j} b} \approx 2 \sum_{j=0}^{12}(-1)^{j} \mathrm{e}^{-\gamma_{j} b}
$$

Moreover, the values of the $k_{j}$ s converge rapidly, and both $\theta_{j}$ and $\gamma_{j}$ are monotonically increasing and tend to $\infty$, which ensure that the term $\mathbb{E}\left[\mathrm{e}^{-\left(2 \gamma_{n}-\theta_{n}\right) Z_{\tau}} \mathbf{1}_{\{\tau<\infty\}}\right]$ vanishes for large $n$; see Table 5 for more details. 
TABLE 5: Values of $k_{j}, \theta_{j}$, and $\gamma_{j}$ when $\lambda=0.05$ and $c=\frac{1}{50}, \frac{1}{20}$.

\begin{tabular}{|c|c|c|c|c|c|c|c|}
\hline \multicolumn{4}{|c|}{$c=\frac{1}{50}$} & \multicolumn{4}{|c|}{$c=\frac{1}{20}$} \\
\hline$j$ & $k_{j}$ & $\theta_{j}$ & $\gamma_{j}$ & $j$ & $k_{j}$ & $\theta_{j}$ & $\gamma_{j}$ \\
\hline 1 & 0.3057 & 0.0000 & 0.3057 & 1 & 0.2874 & 0.0000 & 0.2874 \\
\hline 2 & 0.3128 & 0.6114 & 0.9242 & 2 & 0.3071 & 0.5748 & 0.8819 \\
\hline 3 & 0.3142 & 1.2370 & 1.5512 & 3 & 0.3109 & 1.1891 & 1.5000 \\
\hline 4 & 0.3148 & 1.8654 & 2.1801 & 4 & 0.3125 & 1.8109 & 2.1234 \\
\hline 5 & 0.3151 & 2.4949 & 2.8100 & 5 & 0.3133 & 2.4359 & 2.7492 \\
\hline 6 & 0.3153 & 3.1251 & 3.4404 & 6 & 0.3139 & 3.0625 & 3.3764 \\
\hline 7 & 0.3155 & 3.7557 & 4.0712 & 7 & 0.3142 & 3.6903 & 4.0046 \\
\hline 8 & 0.3156 & 4.3866 & 4.7022 & 8 & 0.3145 & 4.3188 & 4.6333 \\
\hline 9 & 0.3156 & 5.0177 & 5.3334 & 9 & 0.3147 & 4.9478 & 5.2626 \\
\hline 10 & 0.3157 & 5.6490 & 5.9647 & 10 & 0.3149 & 5.5773 & 5.8922 \\
\hline 11 & 0.3157 & 6.2804 & 6.5961 & 11 & 0.3150 & 6.2070 & 6.5221 \\
\hline 12 & 0.3158 & 6.9119 & 7.2277 & 12 & 0.3151 & 6.8371 & 7.1522 \\
\hline
\end{tabular}

TABLE 6: Simulated mean boundary crossing probabilities and their variances (in parentheses) of Corollary 1.

\begin{tabular}{rrccl}
\hline \multirow{2}{c}{$c$} & $b$ & \multicolumn{3}{c}{$\lambda$} \\
\cline { 3 - 5 } & & 0.05 & 0.1 & \multicolumn{1}{c}{1} \\
\hline$\frac{1}{50}$ & 1 & $0.9588(0.000038)$ & $0.9127(0.000091)$ & $0.4857(0.000227)$ \\
& 5 & $0.4199(0.000215)$ & $0.2211(0.000159)$ & $0.0015(0.000002)$ \\
& 10 & $0.0926(0.000085)$ & $0.0269(0.000027)$ & $0.0000(0.000000)$ \\
& 15 & $0.0209(0.000018)$ & $0.0023(0.000002)$ & $0(0)$ \\
& 20 & $0.0039(0.000004)$ & $0.0003(0.000000)$ & $0(0)$ \\
\hline$\frac{1}{20}$ & 1 & $0.9511(0.000040)$ & $0.9209(0.000088)$ & $0.4722(0.000198)$ \\
& 5 & $0.4587(0.000276)$ & $0.2465(0.000238)$ & $0.0016(0.000002)$ \\
& 10 & $0.1182(0.000105)$ & $0.0256(0.000028)$ & $0.0000(0.000000)$ \\
& 15 & $0.0292(0.000031)$ & $0.0031(0.000004)$ & $0(0)$ \\
& 20 & $0.0063(0.000006)$ & $0.0002(0.000000)$ & $0(0)$ \\
\hline$\frac{1}{10}$ & 1 & $0.9746(0.000024)$ & $0.9335(0.000056)$ & $0.4816(0.000257)$ \\
& 5 & $0.5538(0.000185)$ & $0.2881(0.000203)$ & $0.0028(0.000002)$ \\
& 10 & $0.1736(0.000159)$ & $0.0451(0.000044)$ & $0.0000(0.000000)$ \\
& 15 & $0.0511(0.000036)$ & $0.0056(0.000005)$ & $0(0)$ \\
& 20 & $0.0103(0.000009)$ & $0.0006(0.000001)$ & $0(0)$ \\
\hline
\end{tabular}

2.3.2. Monte Carlo simulation results. In Table 6 we present the corresponding simulated results for the number of times a standard Brownian motion crosses one of the boundaries over 1000 simulations. Again, we present the averaged results of 100 repeated calculations. The results are close to those in Table 5.

\section{Conclusions}

By deriving the unconditional boundary crossing probabilities for a standard Brownian motion with stochastic boundaries, we demonstrated how the method of applying the martingale tools allows us to obtain a simple expression. Given the rise of liquidity risk in largevalued interbank payment systems due to the reform, we concentrated on the problem of 
bilateral/multilateral limits that exist in the current UK payment system, CHAPS. The boundary crossing probabilities for a standard Brownian motion with symmetric stochastic process boundaries were studied.

\section{Acknowledgement}

We would like to thank the anonymous referee for many useful comments and suggestions.

\section{References}

[1] Abundo, M. (2002). Some conditional crossing results of Brownian motion over a piecewise-linear boundary. Statist. Prob. Lett. 58, 131-145.

[2] Anderson, T. W. (1960). A modification of the sequential probability ratio test to reduce the sample size. Ann. Math. Statist. 31, 165-197.

[3] Bech, M., Preisig, C. And SoramäKI, K. (2008). Global trends in large-value payments. Econom. Policy Rev. 14, 59-81.

[4] Breiman, L. (1968). Probability. Addison Welsley, Reading, MA.

[5] Brown, R. L., Durbin, J. and Evans, J. M. (1975). Techniques for testing the constancy of regression relationships over time. J. R. Statist. Soc. B 37, 149-192.

[6] CHE, X. (2012). Markov typed models for large-valued interbank payment systems. Doctoral Thesis, London School of Economics.

[7] Che, X., And Dassios, A. (2011). Asymetric linear and stochastic boundary crossing probabilities of Brownian paths. Working paper, London School of Economics.

[8] Doob, J. L. (1949). Heuristic approach to Kolmogorov-Smirnov theorems. Ann. Math. Statist. 20, $393-403$.

[9] Escribá, L. B. (1987). A stopped Brownian motion formula with two sloping line boundaries. Ann. Prob. 15, $1524-1526$.

[10] Frishling, V., Antic, A., Kuchera, A. And Rider, P. (1997). Pricing barrier options with time-dependent drift, volatility and barriers. Working paper, Commonwealth Bank of Australia.

[11] Hall, W. J. (1997). The distribution of Brownian motion on linear stopping boundaries. Sequent. Anal. 16, $345-352$.

[12] Krämer, W., Ploberger, W. and Alt, R. (1988). Testing for structural change in dynamic models. Econometrica 56, 1355-1369.

[13] Novikov, A., Frishling, V. and Kordzakhia, N. (1999). Approximations of boundary crossing probabilities for a Brownian motion. J. Appl. Prob. 36, 1019-1030.

[14] Novikov, A., Frishling, V. And Kordzakhia, N. (2003). Time-dependent barrier options and boundary crossing probabilities. Georgian Math. J. 2, 325-334.

[15] Ricciardi, L. M. (1977). Diffusion Processes and Related Topics in Biology (Lecture Notes Biomath. 14). Springer, Berlin.

[16] Robbins, H. (1970). Statistical methods related to the law of the iterated logarithm. Ann. Math. Statist. 41, 1397-1409.

[17] Robbins, H. And Siegmund, D. (1973). Statistical tests of power one and the integral representation of solutions of certain partial differential equations. Bull. Inst. Math. 1, 93-120.

[18] Roberts, G. O. and Shortland, C. F. (1997). Pricing barriers options with time-dependent coefficients. Math. Finance 7, 83-93.

[19] SEN, P. K. (1981). Sequential Nonparametrics: Invariance Principles and Statistical Inference. John Wiley, New York.

[20] Scheike, T. H. (1992). A boundary-crossing result for Brownian motion. J. Appl. Prob. 29, 448-453.

[21] Siegmund, D. (1985). Sequential Analysis: Tests and Confidence Intervals. Springer, New York.

[22] Siegmund, D. (1986). Boundary crossing probabilities and statistical applications. Ann. Statist. 14, $361-404$.

[23] ShIRYAev, A. N. (2007). On martingale methods in the boundary crossing problems of Brownian motion. Sovrem. Probl. Mat. 8, 3-78 (in Russian).

[24] Wang, L. And Pötzelberger, K. (1997). Boundary crossing probability for Brownian motion and general boundaries. J. Appl. Prob. 34, 54-65. 